\title{
Acquired elastotic hemangioma: A diagnosis to keep in mind
}

\section{Sarra Ben Rejeb', Ines Chelly', Alia Zehani', Beya Chelly', Slim Haouet', Mourad Mokni², Nidhameddine Kchir ${ }^{1}$}

${ }^{1}$ Pathology Department, Rabta Hospital, Bab saadoun, Tunisia, ${ }^{2}$ Dermatology Department, Rabta Hospital, Bab saadoun, Tunisia

Corresponding author: Dr. Sarra Ben Rejeb, E-mail: sarrabenrejeb88@yahoo.fr

\begin{abstract}
Acquired elastotic hemangioma is a relatively newly described cutaneous lesion that presents a characteristic clinicopathologic feature and which should be distinguished from other cutaneous vascular proliferations. Only 10 cases have been reported in literature. We herein describe another case of acquired elastotic hemangioma occurring in the cheek of a 64 year old woman.
\end{abstract}

Key words: Elastosis; Hemangioma; Basal cell carcinoma; Vascular

\section{INTRODUCTION}

Acquired elastotic hemangioma is a recently identified variant of hemangioma, firstly reported in 2002 by Requena et al [1]. Characteristically it is described as a slow growing, solitary erythematous, well defined and asymptomatic plaque which usually develops on sun damaged skin of upper extremities lesion. We herein report another case of acquired elastotic hemangioma occurring of the face.

\section{CASE REPORT}

A 64-year-old woman presented with an asymptomatic, erythematous slowly growing nodule of the cheek. Her family and medical history were unremarkable. Physical examination revealed erythematous, well-defined, non tender, slightly elevated, non blanching plaques of the right cheek. It measured $0.7 \mathrm{~cm}$ in diameter. The suggested clinical diagnosis was basal cell carcinoma. Local surgical excision of the lesion was performed. Histopathologic examination revealed a band-like proliferation of capillary blood vessels involving mainly the superficial dermis and arranged parallel to the epidermis. No cytologic atypia or mitotic figures were seen. The neoformed capillaries were surrounded or intermingled with collagen bundle showing intense solar elastosis (Figs. 1 - 3). Inflammation was absent. On immunohistochemichemistry, the neoplastic endothelial cells of the neoformed capillaries were strongly positive for CD34 (Fig. 4). No staining with HHV8 was found. A peripheral ring of actin-positive (Alpha smooth muscle) pericytes were observed (Fig. 5).

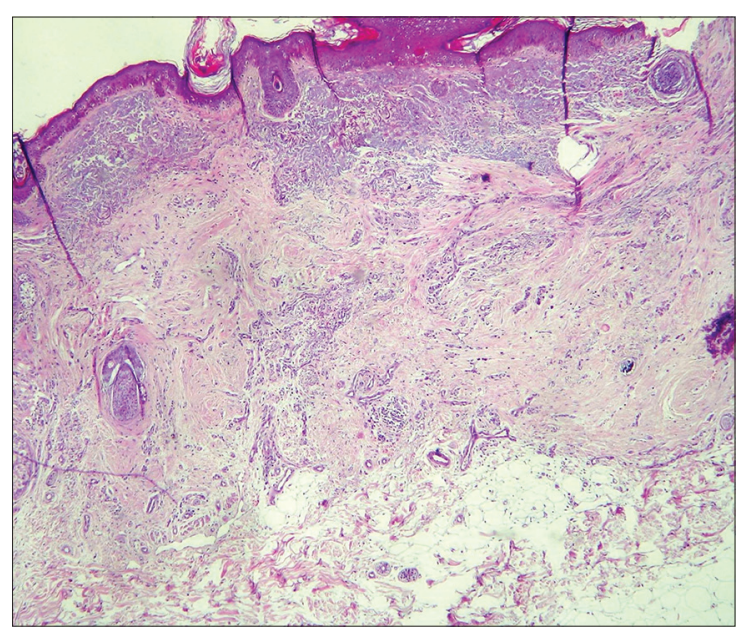

Figure 1: Band-like proliferation of capillary blood vessels intermingled with intense solar elastosis (HE x 100).

\footnotetext{
How to cite this article: Ben Rejeb S, Chelly I, Zhani A, Chelly B, Haouet S, Mokni M, Kchir N.. Acquired elastotic hemangioma: A diagnosis to keep in mind. Our Dermatol Online. 2017;8(4):443-445.

Submission: 02.01.2017; Acceptance: 10.03.2017

DOI: 10.7241 /ourd.20174.125
} 


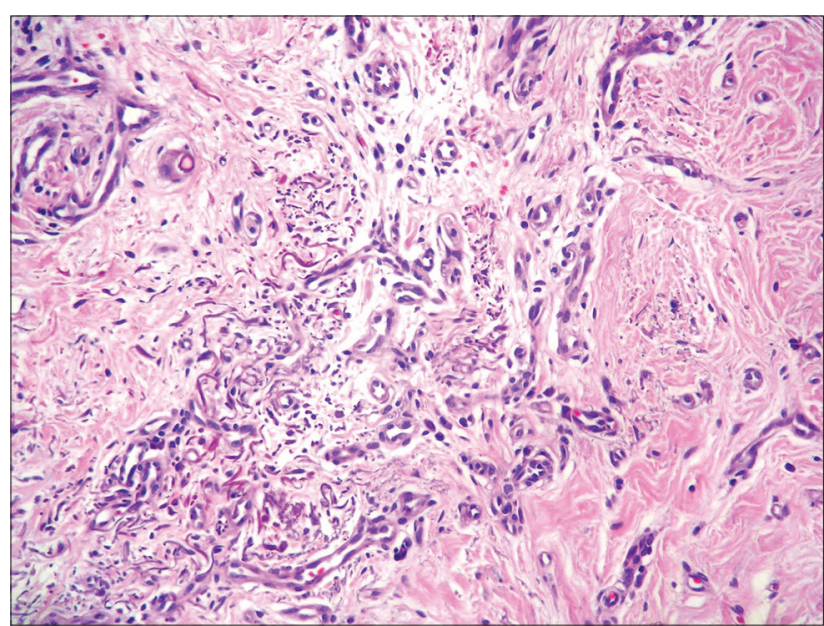

Figure 2: Proliferation of small blood vessels bordered with regular endothelial cells and surrounded by collagen bundle showing intense solar elastosis (HE x 200).

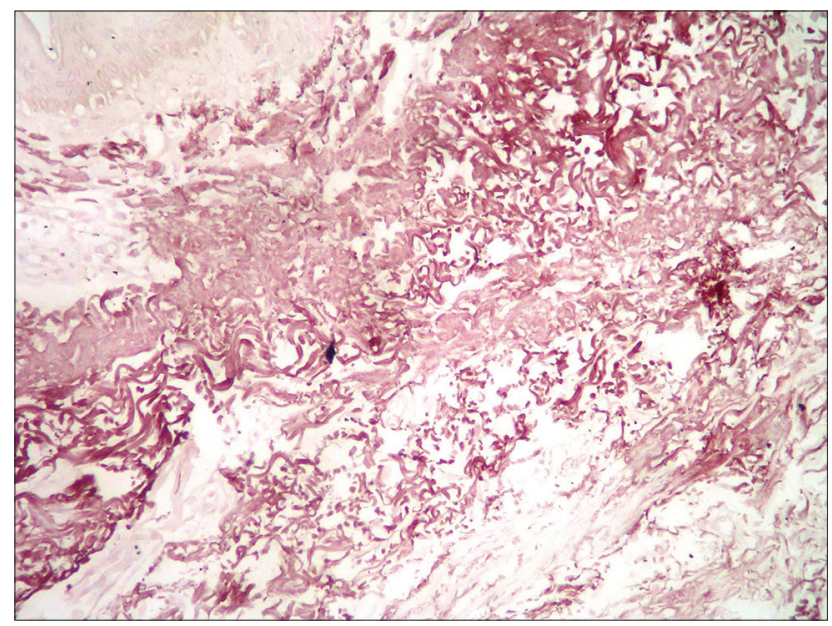

Figure 3: Elastosic collagen bundles (orcein coloration X 200).

The diagnosis of acquired elastotic hemangioma was favored.

\section{DISCUSSION}

Acquired elastotic hemangioma (AEH) is a recently proposed entity described by Requena et al [1] and since there only 10 cases have been reported in litterature. A distinctive clinicopathological presentation allowed the authors to consider this condition as a separate entity [1-2]. This lesion classically occurs in middle aged or elderly women with the mean age of 64 years. Clinically, it presents as an irregularly shaped, slowly growing, well defined, non-blanching, erythematous to violaceous plaque, ranging from 2 to $5 \mathrm{~cm}$ in diameter [1]. Generally the lesions are asymptomatic, but occasionally can be painful. They show a strong predilection for the extensor surfaces of the forearms,

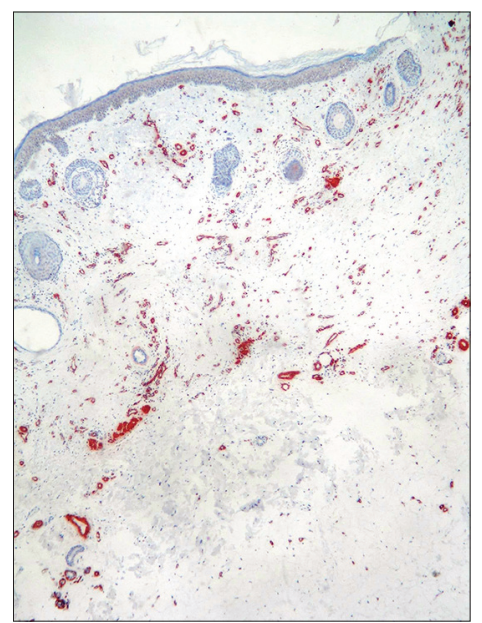

Figure 4: Positive staining of endothelial neoplastic cells for CD34 (IHC x200).

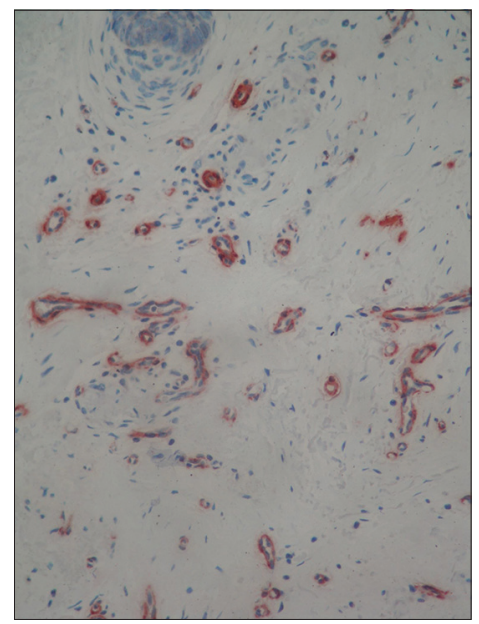

Figure 5: Positive staining of pericytes for alpha-smooth muscle (IHC $x$ 400).

but may also be found on the lower lip, shoulder, nose, and neck [2]. Clinically, aquired elastotic hemangioma may be confused with a superficial basal cell or Bowen's disease [1]. Histologically, the classic finding is a band-like proliferation of capillary blood vessels arranged parallel to the epidermis and confined to the superficial dermis [3]. The vessels contain well formed round or elongated lumens lined by a single layer of monomorphous endothelial cells without any atypia or mitosis surrounded with a layer of pericytes. The capillary proliferation is separated from the epidermis by a zone of non involved papillary dermis. The epidermis is unremarkable or atrophic. The most attractive feature is the intense accompanying solar elastosis which is surrounding or intermingled with the capillaries [1-3]. Immunohistochemical studies demonstrated the endothelial nature of the neoplastic cells which are usually positive for CD31 
and CD34 markers. The capillaries have a peripheral ring of actin-positive pericytes [1]. Acquired elastotic hemangioma was initially thought to be a true vascular tumor, however research has recently proposed a lymphatic origin after noting expression of D2-40 [2]. Proliferating markers Ki-67 and MPM2 stain only a few nuclei of the endothelial cells of the vessels $[1,4]$. Histopathologic differential diagnoses of AEH include cutaneous capillary proliferations that develop during adulthood, namely Mali's acroangiodermatitis, cherry angioma, acquired tufted hemangioma, hobnail hemangiomas and early Kaposi's sarcoma with a predominant angiomatous pattern. Kaposi's sarcoma histologically exhibits jagged, vascular spaces lined by thin endothelial cells with a lymphoplasmacytic infiltrate, red blood cell extravasation and positive staining for HHV8. An acquired tufted angioma shows a "cannon-ball" histopathological pattern with lobules of capillary tufts scattered in the dermis and subcutaneous fat. A targetoid hemosiderotic hemangioma displays dilated vascular spaces in the superficial dermis, lined by prominent hobnail endothelial cells and anastomosing collagen bundles with hemosiderin deposits [1]. The band-like capillaries arranged along the superficial dermis with solar elastosis is a unique feature characterizing acquired elastotic hemangioma. The etiology is still unknown, but the finding of solar elastosis supports the role of sun damage as an inciting cause. In most cases, there is no history of previous trauma. In all reported cases, acquired elastotic hemangioma behaves in a benign fashion [1-4].

\section{CONCLUSION}

Acquired elastotic hemangioma is a benign, asymptomatic lesion which commonly occurs on sun damaged skin. Treatment is unnecessary, but because of its misleading clinical presentation, a surgical excision is usually performed.

\section{Consent}

The examination of the patient was conducted according to the Declaration of Helsinki principles. Written informed consent was obtained from the patient for publication of this article.

\section{REFERENCES}

1. Requena L, Kutzner H, Mentzel T. Acquired elastotic hemangioma: A clinicopathologic variant of hemangioma. J Am Acad Dermatol. 2002;47:371-7.

2. Martorell-Calatayud, A, Balmer N, Sanmartin O, Diaz-Recuero JL, Sangueza OP. Definition of the features of acquired elastotic hemangioma reporting the clinical and histopathological characteristics of 14 patients. J Cutan Pathol. 2010;37:460-4.

3. Goh SGN, Calonje E. Cutaneous vascular tumors: an update. Histopathology. 2008;52:661-73.

4. Tong PL, Beer TW. Acquired elastotic hemangioma: ten cases with immunohistochemistry refuting a lymphatic origin in most lesions. J Cutan Pathol. 2010;37:1259-60.

Copyright by Sarra Ben Rejeb, et al. This is an open access article distributed under the terms of the Creative Commons Attribution License, which permits unrestricted use, distribution, and reproduction in any medium, provided the original author and source are credited.

Source of Support: Georgia Dermatopathology Associates, Atlanta, Georgia, USA, Conflict of Interest: None declared. 